\title{
Classification of schizophrenia using feature-based morphometry
}

\author{
U. Castellani $\cdot$ E. Rossato $\cdot$ V. Murino $\cdot$ \\ M. Bellani - G. Rambaldelli • C. Perlini • \\ L. Tomelleri $\cdot$ M. Tansella $\cdot$ P. Brambilla
}

Received: 3 December 2010/Accepted: 16 July 2011/Published online: 9 September 2011

(C) Springer-Verlag 2011

\begin{abstract}
The objective of this study was to use a combined local descriptor, namely scale invariance feature transform (SIFT), and a non linear support vector machine (SVM) technique to automatically classify patients with schizophrenia. The dorsolateral prefrontal cortex (DLPFC), considered a reliable neuroanatomical marker of the disease, was chosen as region of interest (ROI). Fifty-four schizophrenia patients and 54 age- and gender-matched normal controls were studied with a $1.5 \mathrm{~T}$ MRI (slice thickness $1.25 \mathrm{~mm})$. Three steps were conducted: (1) landmark detection and description of the DLPFC, (2) feature vocabulary construction and Bag-of-Words (BoW) computation for brain representation, (3) SVM classification which adopted the local kernel to implicitly implement
\end{abstract}

The preliminary results of this study were presented at the XI Conference of the Italian Association for Artificial Intelligence (AIxIA), Reggio Emilia, Italy, December 9-12, 2009. The dataset used in this work is part of a larger database kept by the Research Unit on Brain Imaging and Neuropsychology (RUBIN) at the Department of Public Health and Community Medicine-Section of Psychiatry and Clinical Psychology of the University of Verona.

U. Castellani · E. Rossato · V. Murino

Department of Computer Science,

University of Verona, Verona, Italy

V. Murino

Istituto Italiano di Tecnologia, Genoa, Italy

M. Bellani · G. Rambaldelli · C. Perlini · L. Tomelleri ·

M. Tansella

Department of Public Health and Community Medicine,

Section of Psychiatry and Clinical Psychology,

Inter-University Centre for Behavioural Neurosciences,

University of Verona, Verona, Italy

P. Brambilla

IRCCS "E. Medea" Scientific Institute, Udine, Italy the feature matching. Moreover, a new weighting approach was proposed to take into account the discriminant relevance of the detected groups of features. Substantial results were obtained for the classification of the whole dataset (left side $75 \%$, right side $66.38 \%$ ). The performances were higher when females (left side $84.09 \%$, right side $77.27 \%$ ) and seniors (left side $81.25 \%$, right side $70.83 \%$ ) were considered separately. In general, the supervised weighed functions increased the efficacy in all the analyses. No effects of age, gender, antipsychotic treatment and chronicity were shown on DLPFC volumes. This integrated innovative ROI-SVM approach allows to reliably detect subjects with schizophrenia, based on a structural brain marker for the disease such as the DLPFC. Such classification should be performed in first-episode patients in future studies, by considering males and females separately.

Keywords Neuroimaging $\cdot$ MRI $\cdot$ Support vector machine $\cdot$ Dorsolateral prefrontal cortex $\cdot$ Shape morphometry

\section{P. Brambilla}

Department of Experimental Clinical Medical Sciences, Inter-University Centre for Behavioural Neurosciences (ICBN), University of Udine, Udine, Italy

P. Brambilla $(\bowtie)$

Department of Experimental Clinical Medical Sciences,

University of Udine, P.le Kolbe 3, 33100 Udine, Italy

e-mail: paolo.brambilla@uniud.it 


\section{Introduction}

Computational neuroanatomy using magnetic resonance imaging (MRI) has been used consistently in schizophrenia to detect specific morphological abnormalities of the brain in comparison with normal subjects (Giuliani et al. 2005). In general two methods are routinely used, i.e. the region of interest (ROI) analysis or the voxel-based morphometry (VBM) (Ashburner and Friston 2000). The ROI methods are focussed on specific brain regions which are manually traced by expert operators. The VBM considers the whole brain after a normalization procedure which maps the current brain onto a standard reference, namely the stereotaxic space, allowing a voxel-by-voxel comparison. Both methods have reported some consistent findings such as cortical atrophy, particularly of the prefrontal cortex, with related ventricular enlargement, shrinkage of the hippocampus and of the superior temporal gyrus, and reduction of the cerebral asymmetry (Andreone et al. 2007a; Arnone et al. 2008; Bellani et al. 2010a; Fornito et al. 2009; Kempton et al. 2010). However, neither technique enables patients with schizophrenia to be classified automatically, based on the brain's features. In this perspective, apart from standard volumetric methods (Ashburner and Friston 2000; Baiano et al. 2008), few studies have applied innovative approaches to detect schizophrenia based on the brain characteristics (Fan et al. 2007; Gerig et al. 2001; Koutsouleris et al. 2009; Yoon et al. 2007). Specifically, Gerig et al. (2001) proposed a ROI-based morphometric analysis by defining spherical harmonics and a 3D skeleton as shape descriptors, i.e. the shape descriptor-based approach was successfully compared to classical volumetric techniques. Yoon et al. (2007) utilized a support vector machine (SVM) to classify cortical thickness, which was measured by calculating the Euclidean distance between linked vertices on the inner and outer cortical surfaces. Fan et al. (2007) combined deformation-based morphometry with SVM, capturing multivariate relationships across various anatomical regions. Finally, Koutsouleris et al. (2009) performed a whole brain SVM analysis, detecting the dimensionality of MR images by the optimal number of uncorrelated principal components obtained by principal component analysis (PCA). Several cortical and subcortical areas across the two hemispheres were found to characterize patients with schizophrenia, at-risk subjects and unaffected family members (Davatzikos et al. 2005; Fan et al. 2007, 2008; Koutsouleris et al. 2009; Yoon et al. 2007). However, with the exception of the preliminary study by Gerig et al. (2001), these reports were not driven by a priori hypothesis and did not consistently detect any specific structural markers. Also, they applied multivariate whole brain techniques, thus being limited by the analysis of an immense dimensional space in relatively small samples.

In this study, we aimed at automatically classifying schizophrenia by applying a ROI-based machine learning approach (Duda et al. 2001) within the dorsolateral prefrontal cortex (DLPFC), a reliable structural marker for schizophrenia (Potkin et al. 2009; Prasad et al. 2007; Yoon et al. 2008). This technique utilizes a few, significant landmarks to detect and characterise local region descriptors. The novelty therefore consists in characterising brain abnormalities in terms of intra-ROI local patterns which are not necessarily spatially coherent, using consistent neuroanatomical features for the disease. The underlying hypothesis consists in relaxing the common constraint that morphological anomalies appear at the same voxel location for the entire population. Therefore, a new kernel of a SVM was designed to compare a pair of brains represented by an unordered set of features. The proposed method is inspired by the Bag-of-Words (BoW) (Cruska et al. 2004) paradigm which implicitly implements feature matching within the SVM framework (Grauman and Darrell 2007). Finally, a weighting function was introduced to define the relevance of the detected features, namely the visual words, in discriminating between patients and controls.

\section{Materials and methods}

\section{Sample}

Fifty-four patients suffering from schizophrenia as defined by the Diagnostic and Statistical Manual of Mental Disorders, 4th edition, (DSM-IV) (American Psychiatric Association 1994) were recruited from the South Verona Psychiatric Case Register (Amaddeo et al. 1997, 2009) (Table 1). The register includes information about patients residing in the epidemiologically defined catchment area of South Verona (with a population of approximately 100,000 inhabitants) and treated by the South Verona Communitybased Mental Health Service (CMHS) and related clinics. Diagnostic evaluation was based on the Item Group Checklist of the Schedule for Clinical Assessment in Neuropsychiatry (IGC-SCAN) (World Health Organisation 1992). These assessments were conducted by trained clinical psychologists with extensive experience of using the SCAN, as previously described (Andreone et al. 2007b), who were blind to diagnosis. The Italian version of the SCAN was edited by our group (World Health Organisation 1996) and our investigators attended specific courses held by official trainers on how to administer this scale. The inter- and intra-rater reliability of the IGC-SCAN assessments was monitored by regular quality control meetings. Diagnostic validity was further confirmed by clinical consensus by two qualified psychiatrists. The patients' psychopathology was rated using the Brief Psychiatric Rating Scale (BPRS, 24-item version) (Ventura et al. 2000). Information about age of onset, duration of 
Table 1 Demographic features of the subjects

\begin{tabular}{lll}
\hline & $\begin{array}{l}\text { Healthy controls } \\
(N=54)\end{array}$ & $\begin{array}{l}\text { Patients with } \\
\text { schizophrenia }(N=54)\end{array}$ \\
\hline $\begin{array}{l}\text { Age (years) } \\
\begin{array}{l}\text { Gender (females/ } \\
\text { males) }\end{array}\end{array}$ & $\begin{array}{l}39.19 \pm 10.05 \\
\text { Race }\end{array}$ & $\begin{array}{l}37.96 \pm 10.90 \\
19 / 35\end{array}$ \\
$\begin{array}{l}\text { Duration of illness } \\
\quad \text { years) }\end{array}$ & Caucasian & Caucasian \\
$\begin{array}{l}\text { AP Lifetime } \\
\text { treatment (years) }\end{array}$ & $12.53 \pm 10.04$ \\
$\begin{array}{l}\text { CPZ-equivalent } \\
\text { dose (mg) }\end{array}$ & $10.77 \pm 9.23$ \\
BPRS total scores & & $241.91 \pm 176.29$ \\
\hline
\end{tabular}

Healthy controls and patients with schizophrenia did not significantly differ for age or gender $(p>0.05)$

$A P$ antipsychotic, $C P Z$ chlorpromazine

illness, and number of hospital admissions was obtained during an interview and from medical records. Fifty-four matched healthy individuals without any personal lifetime history of DMS-IV Axis I disorders, recruited from the same catchment area, were also studied (Table 1). Exclusion criteria for all participants were (a) alcohol or substance abuse within the preceding 6 months, as defined by the DSM-IV (b) any current major medical or neurological illness, (c) history of traumatic head injury with loss of consciousness, (d) DSM-IV axis I comorbidity. Additional exclusion criteria for comparator subjects were (a) any selfreported history of psychiatric disorders in first-degree relatives (b) any prescribed medication.

The study was approved by the Ethics Committee of the Azienda Ospedaliera of Verona. All the participants provided their signed informed consent, having understood the nature and purpose of the study after it was explained to them.

Magnetic resonance imaging acquisition

The MRI scans were acquired using a $1.5 \mathrm{~T}$ Siemens Magnetom Symphony Maestro Class, Syngo MR 2002B. All the participants were provided with earplugs to reduce acoustic noise and their head was placed in a comfortable head holder to keep it steady in order to minimize movement artefacts. Initially, exploratory T1-weighted images $\left(\mathrm{TR}=450 \mathrm{~ms}, \mathrm{TE}=14 \mathrm{~ms}\right.$, flip angle $=90^{\circ}, \mathrm{FOV}=$ $230 \times 230$, slice thickness $=5 \mathrm{~mm}$, matrix size $=384 \times$ 512 ) were obtained to verify the subject's head position and the quality of the image. A coronal 3D MPR sequence was acquired $\left(\mathrm{TR}=2,060 \mathrm{~ms}, \mathrm{TE}=3.9 \mathrm{~ms}\right.$, flip angle $=15^{\circ}$, $\mathrm{FOV}=176 \times 235$, slice thickness $=1.25 \mathrm{~mm}$, matrix size $=270 \times 512, \quad \mathrm{TI}=1,100)$ to obtain 144 images covering the entire brain.
Automatic classification analysis

The proposed method was based on three main steps: (1) landmark detection and description, (2) feature vocabulary construction and computation, (3) SVM classification.

\section{Landmark point detection and description}

A landmark or feature is a set of points that can be clearly differentiated from its neighbouring image points. In this paper, we employed the Difference of Gaussians (DoG) point detector (Lowe 2004) which is robust to imagetranslation, -rotation and -scale. This implies that a landmark can be detected without requiring an explicit registration procedure. Moreover, the region of influence of each landmark (i.e., the neighbourhood) was also estimated with this technique. In general, a wider region was adaptively defined for homogeneous areas and viceversa. Therefore, for each landmark the scale invariance feature transform (SIFT) descriptor (Lowe 2004) was applied to characterize its local neighbourhood. In practice, the pixels of the landmark's neighbourhood are encoded into a multidimensional feature vector which effectively and concisely describes the local area. A pair of successive slices from the DLPFC, with the extracted landmarks and their region of influence are reported in Fig. 1. Slice thickness of $1.25 \mathrm{~mm}$ was utilized. Then the most characteristic patches in terms of strong local pattern variations were selected from each brain. Here, the main idea was to verify whether there were brain anomalies among those variations.

\section{Feature vocabulary construction}

After the landmark detection was completed, each brain was represented by a set of unordered feature vectors. Moreover, such sets generally appeared with different cardinalities. In order to compare a pair of brains, the Bagof-Words (BoW) approach was introduced (Cruska et al. 2004). The set of feature vectors coming from all brains was clusterized by employing the k-means clustering technique (Duda et al. 2001). According to the BoW paradigm, the centroids of the clustering were referred as visual words or feature prototypes (Cruska et al. 2004). Indeed, the set of visual words provides a quantization of the feature space, i.e., the so called feature vocabulary. Then, each set of feature vectors observed on each brain was transformed into a histogram that counted the frequency of occurrence of such feature prototypes (Cruska et al. 2004). In order to obtain this, each feature vector of a brain was compared with all the visual words and was associated to the closest one. The outputted histogram became the descriptor of the brain. The set of landmark points of the DLPFC extracted from the whole dataset is 


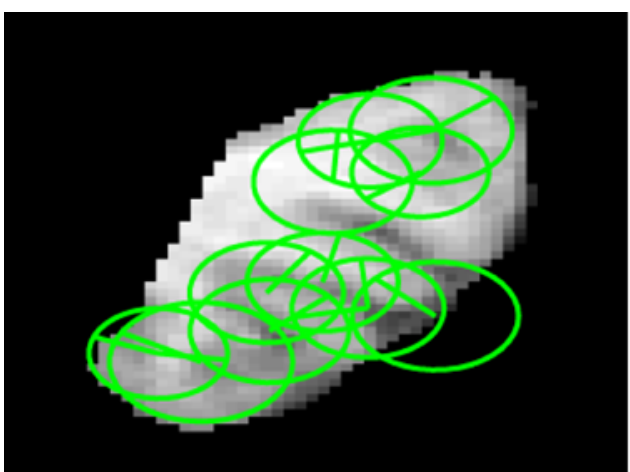

Fig. 1 Two succeeding slices from the dorsolateral prefrontal cortex (DLPFC). Landmark points are identified by green ellipsoids and represent feature that can be clearly differentiated from its

shown in Fig. 2. Each landmark is coloured according to its closest visual words. In this fashion, similar landmarks are associated to the same visual word and visualized with the same colour.

The relationship between feature prototypes and morphological abnormalities due to schizophrenia was tentatively captured. For each cluster of features its discriminative relevance was measured by counting the occurrences for each group separately. In particular, the following weighting function was defined for each visual word:

$w_{i}\left(n_{p}^{c_{i}}, n_{c}^{c_{i}}\right)= \begin{cases}1.5 & \text { if }\left\|n_{p}^{c_{i}}-n_{c}^{c_{i}}\right\| \geq \Delta \\ 0.5 & \text { otherwise }\end{cases}$

where $c_{i}$ is the $i$ th centroid (i.e., the visual word, $i=1, \ldots, K$ ), $n_{p}^{c_{i}}$ and $n_{c}^{c_{i}}$ are the percentages of patients and controls in $c_{i}$, and $\Delta$ is a heuristic constant. In this way, clusters composed

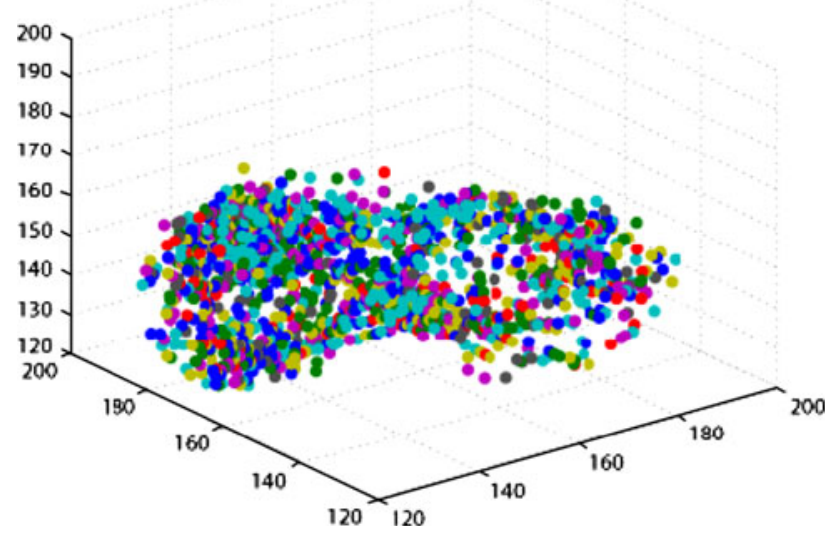

Fig. 2 Clusterized feature vectors are shown. The centroids of the clustering are referred as visual words (or feature prototypes) and each point is coloured according to its visual word. Similar landmarks are associated to the same visual word and therefore are visualized with the same colour

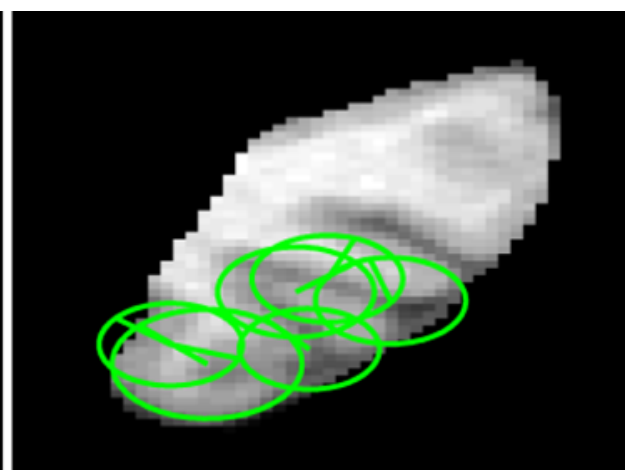

neighbouring image points. The region of influence of a landmark is represented by the set of pixel inside its ellipsoid; in general, a wider region is adaptively defined for homogeneous areas and viceversa

by a clear majority of the population (i.e. patients or controls) were considered to be more discriminant for the classification.

\section{Support vector machine classification}

SVMs (Brurges 1998) are powerful classifiers which have reliably been used for schizophrenia (Fan et al. 2007). Note that usually a SVM requires a fixed length vector which characterizes globally the subject to be classified. Here, however, due to the BoW representation, a subject (i.e., a brain) was encoded by a set of local features. In particular, the novelty consisted in employing a suitable kernel function to implicitly implement the feature matching. Such kernels are generally referred to as local kernels or matching kernels (Grauman and Darrell 2007).

In order to construct a BoW histogram of a new brain, we compared each of the extracted features with the visual words w.r.t. the visual vocabulary by counting the number of features assigned to each visual word. The BoW representation $h^{A}$ for brain $A$ was obtained in this way.

In detail, the kernel function was defined as:

$K\left(h^{A}, h^{B}\right)=\sum_{i=1}^{K} w_{i} \min \left(h_{i}^{A}, h_{i}^{B}\right)$

Where $h_{i}^{A}$ denotes the count of the $i$ th bin of the histogram $h^{A}$ with $K$ bins, and $w_{i}$ is computed from Eq. 1. Such a kernel was called a weighted histogram intersection function and was shown to be a valid kernel (Grauman and Darrell 2007). Histograms were assumed to be normalized such that

$\sum_{i=1}^{K} h_{i}=1$

As observed by Grauman and Darrell (2007), the proposed kernel implicitly encoded the point-to-point 
matching, since corresponding features were likely to belong to the same histogram bin. Indeed, the histogram intersection function counted the number of feature matching, which are intermediated by the visual words. More in detail, since two points are declared as being a 'match' if both are associated to the same visual word, the tolerance of matching (i.e. the maximum error matching of a pair of points) is bounded by the size of cluster associated to the visual word.

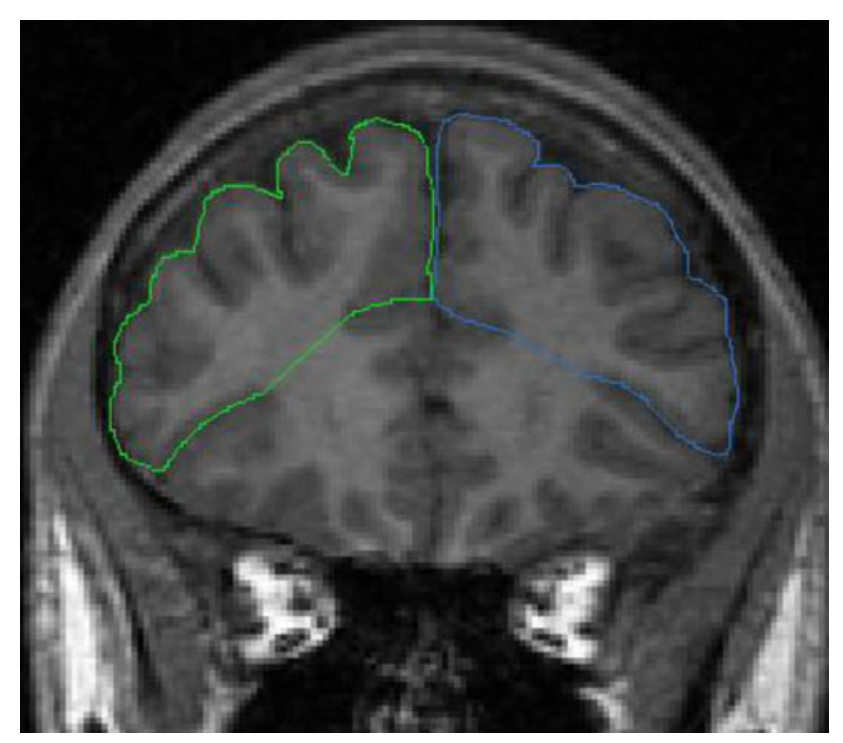

Fig. 3 Dorsolateral prefrontal cortex tracing. The superior border of the DLPFC was the superior frontal sulcus, the inferior border was the upper border of the Sylvian fissure posteriorly and the horizontal ramus of the Sylvian fissure anteriorly; the lateral boundary was the edge of the brain, and the medial boundary was the line connecting the most medial point of the superior frontal sulcus with the Sylvian fissure/horizontal ramus
Dorsolateral prefrontal cortex landmarks

All imaging data were analyzed using the BRAINS2 software developed at the University of Iowa. The superior border of the DLPFC was the superior frontal sulcus, the inferior border was the upper border of the Sylvian fissure posteriorly and the horizontal ramus of the Sylvian fissure anteriorly. The lateral boundary was the edge of the brain, and the medial boundary was the line connecting the most medial point of the superior frontal sulcus with the Sylvian fissure/horizontal ramus (Fig. 3). The tracing started anteriorly to the posterior border of the genu and ended at the anterior border of the horizontal ramus of the Sylvian fissure, as per a previously published technique (Prasad et al. 2005). A rater who was blind to the subjects' identity traced all the scans, after reaching intra-class correlation coefficients (ICCs) $>0.90$ (0.92 for left DLPFC, 0.98 for right DLPFC) with another rater by blindly tracing 10 randomly selected scans. The volumes $(\mathrm{ml})$ were obtained by summing the volumes of all relevant slices and were expressed in $\mathrm{cm}^{3}$. Intracranial volume (ICV) was traced in the coronal place along the border of the brain and included the cerebrospinal fluid, dura mater, sinus, optic chiasma, brainstem, and cerebral and cerebellar matter. The inferior border did not extend below the base of the cerebellum. An inter-rater reliability of 0.97 was achieved for the ICV measurements.

\section{Results}

Landmark extraction was obtained using the SIFT implementation available from http://vision.ucla.edu/vedaldi. Then feature points were properly clusterized in order to obtain the visual words. The Matlab (http://www.math works.com) version 7.4 of the $K$-mean algorithm was used
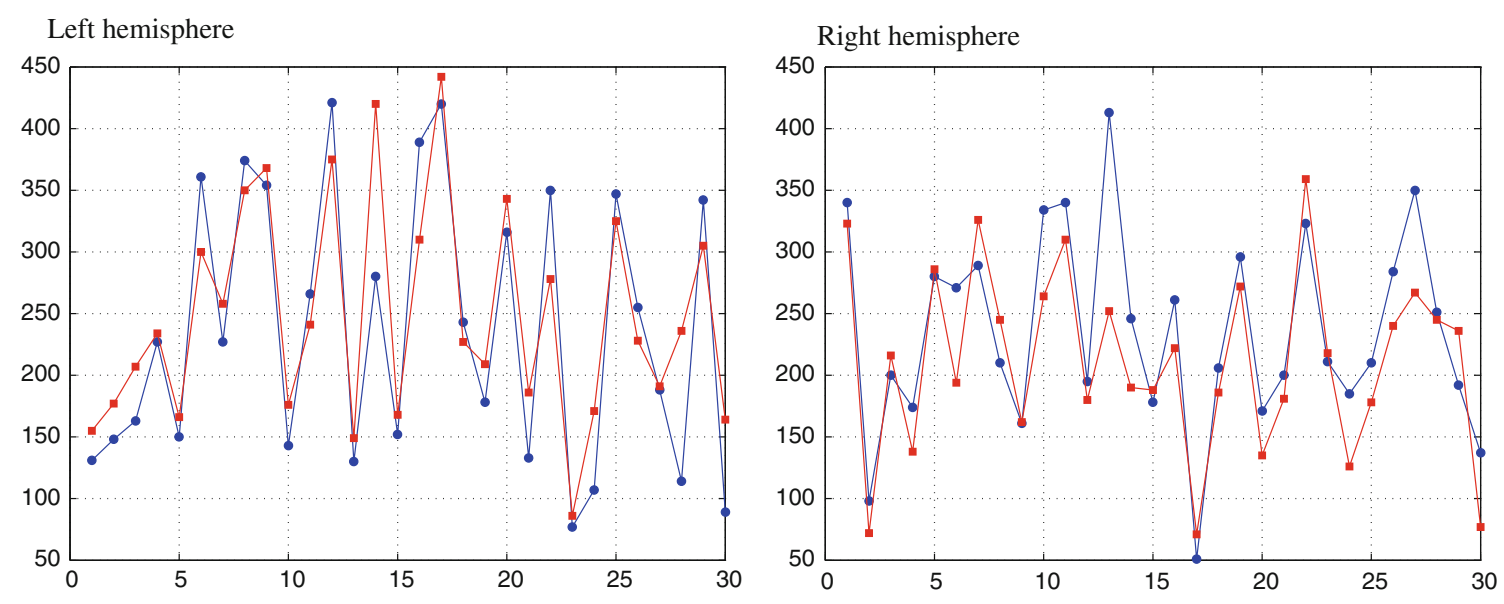

Fig. 4 Histogram of word occurrences for the whole dataset for the left and right hemispheres. Patients with schizophrenia are marked in red, healthy controls in blue 


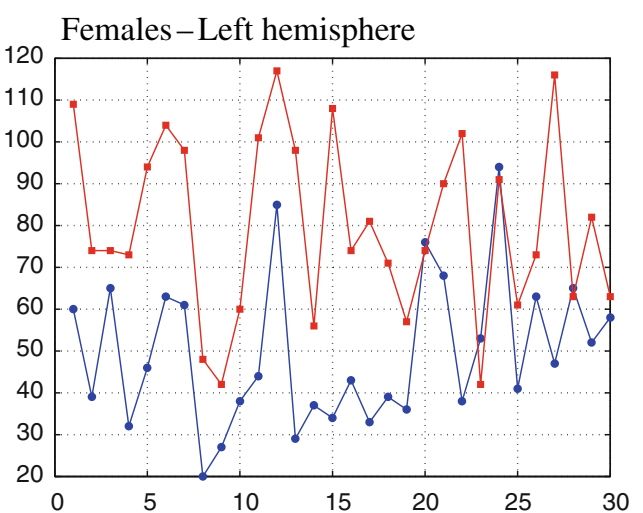

Females-Right hemisphere
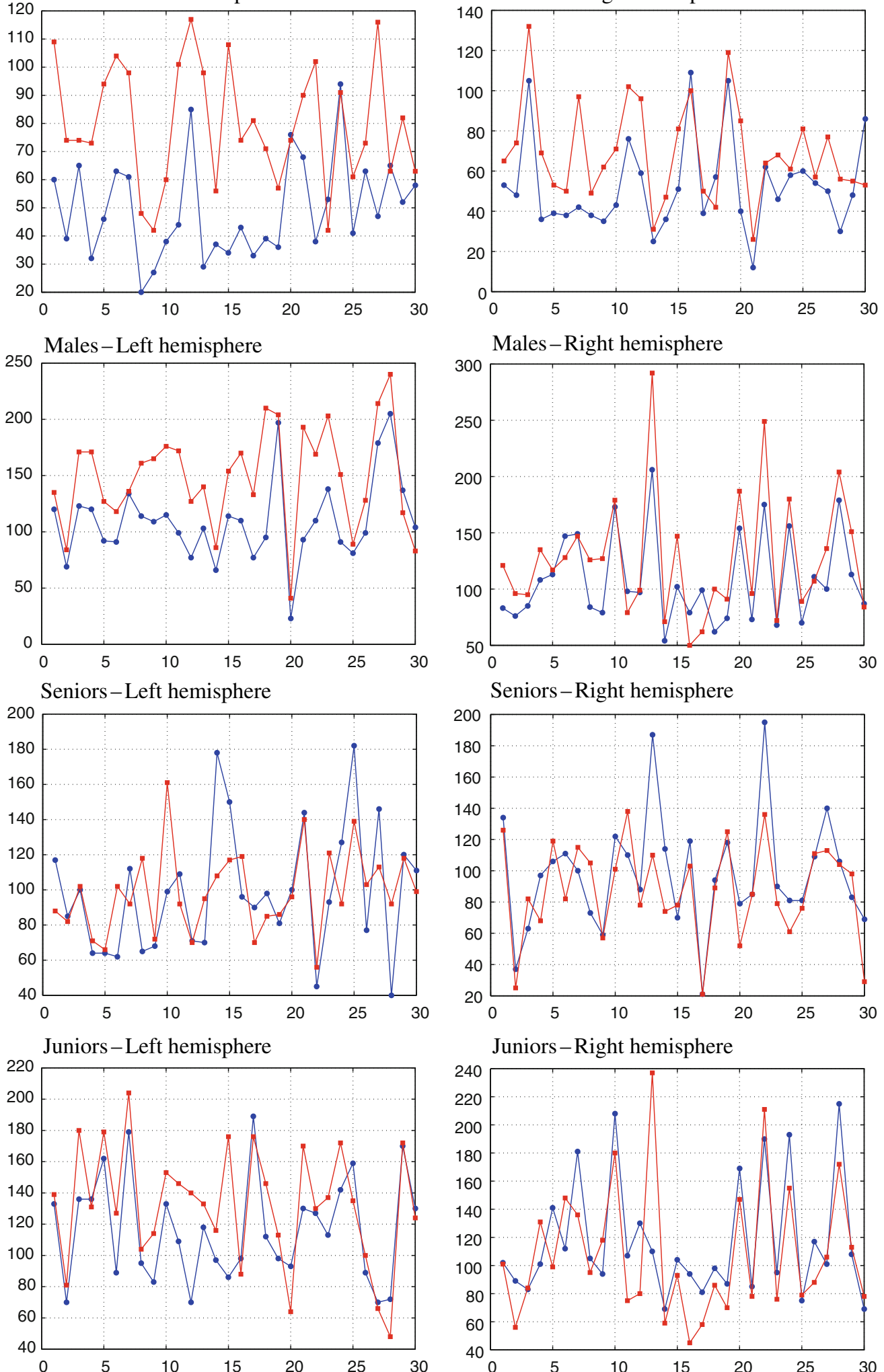

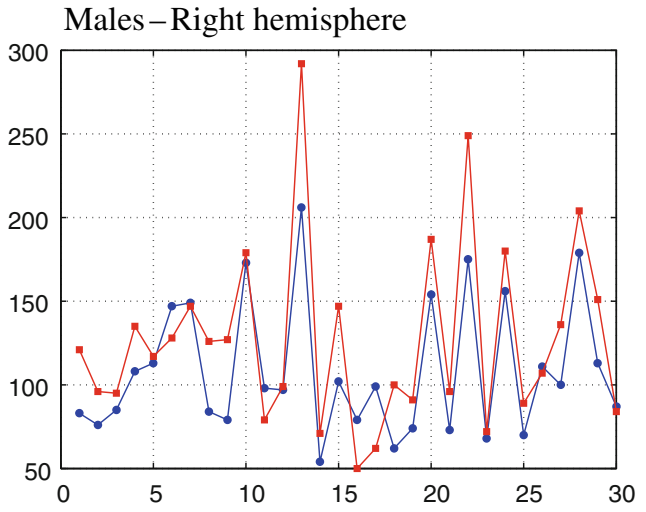

Seniors-Right hemisphere

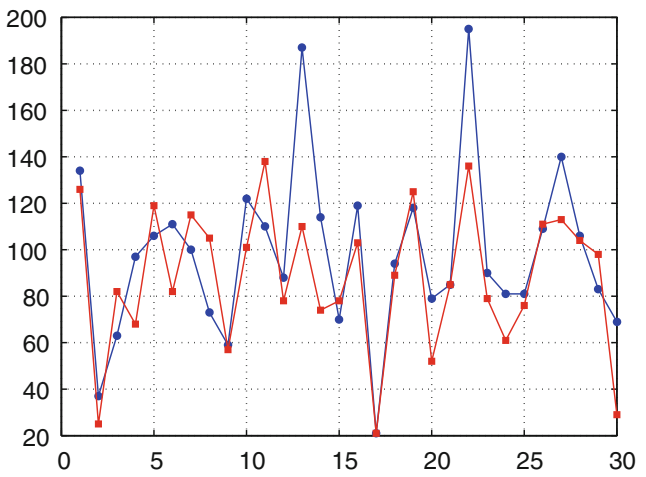

Juniors-Right hemisphere

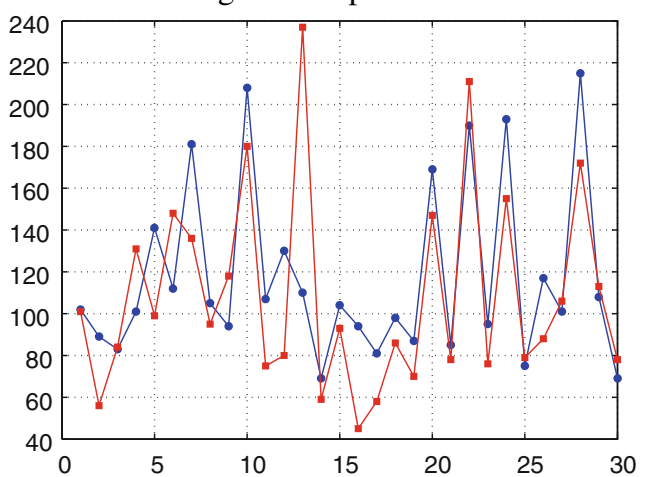

Fig. 5 Word relevance for patients with schizophrenia (red) and healthy controls (blue), stratified by gender and age 
Table 2 Classification rate for healthy controls and patients with schizophrenia

Senior subjects are $\geq 40$ years and juniors are $<40$ years

$n$ number

\begin{tabular}{|c|c|c|c|c|c|c|}
\hline \multirow[t]{2}{*}{ Experiment } & \multirow{2}{*}{$\begin{array}{l}\text { Healthy } \\
\text { controls }(n)\end{array}$} & \multirow{2}{*}{$\begin{array}{l}\text { Patients with } \\
\text { schizophrenia }(n)\end{array}$} & \multicolumn{2}{|l|}{ Left } & \multicolumn{2}{|l|}{ Right } \\
\hline & & & $\begin{array}{l}\text { Weight } \\
\text { score }(\%)\end{array}$ & $\begin{array}{l}\text { Raw } \\
\text { score }(\%)\end{array}$ & $\begin{array}{l}\text { Weight } \\
\text { score }(\%)\end{array}$ & $\begin{array}{l}\text { Raw } \\
\text { score }(\%)\end{array}$ \\
\hline Whole dataset & 54 & 54 & 75.00 & 62.93 & 66.38 & 59.48 \\
\hline Females & 25 & 19 & 84.09 & 77.27 & 77.27 & 72.73 \\
\hline Males & 29 & 35 & 60.00 & 44.62 & 67.69 & 50.77 \\
\hline Seniors & 23 & 25 & 81.25 & 73.52 & 70.83 & 64.12 \\
\hline Juniors & 31 & 29 & 71.67 & 55.27 & 63.33 & 51.18 \\
\hline
\end{tabular}

by fixing $K=30$. Therefore, the relevance of each visual word was computed by obtaining the weighs $w_{i}$ (Fig. 3). Also, in order to take into account the intra-class variability, the whole dataset was stratified by sex (males and females) and age (subjects $<40$ years, and subjects $\geq 40$ years) (Fig. 4, 5).

The classification is shown in Table 2. Scores were obtained by leave-one-out cross validation (Duda et al. 2001). In general, a dramatic improvement was observed when weights were applied. Substantial results were obtained for the classification of the whole dataset (left hemisphere $75 \%$, right hemisphere $66.38 \%$ ). Moreover, performances increased when only females (left side $84.09 \%$, right side $77.27 \%$ ) and only seniors (left side $81.25 \%$, right side $70.83 \%$ ) were taken into consideration.

Interestingly, DLPFC volumes significantly inversely correlated with length of illness on the right side (Spearman's rho coefficient $=-0.43, p=0.001$ ) with a trend for significance on the left one (Spearman's rho coefficient $=-0.23, p=0.10)$. In contrast, no significant correlations were found between antipsychotic lifetime treatment or dosages and bilateral DLPFC volumes (partial correlation analyses controlling for length of illness, $p>0.05)$. Age did not show any significant association in both control and schizophrenia group with DLPFC volumes (Spearman's correlation, $p>0.05$ ). Moreover, DLPFC size did not significantly differ between patients treated with typical and atypical antipsychotics and between chronic and non-chronic schizophrenia patients separated in accordance to mean of length of illness (GLM multivariate with age, gender, and ICV as covariates, $p>0.05)$. Finally, schizophrenia patients did not show any significant differences for DLPFC volumes when males and females (GLM multivariate with age, and ICV as covariates, $\mathrm{p}>0.05)$ and junior and senior individuals were compared (GLM multivariate with gender, and ICV as covariates, $p>0.05)$. Age, length of illness, BPRS scores, and antipsychotic lifetime treatment did not differ between female and male schizophrenia subjects and between patients treated with typical and atypical antipsychotic drugs (student $t$ test, $p>0.05$ ).

\section{Discussion}

This study showed that our innovative approach integrating ROI and SVM techniques allows to consistently classify subjects with schizophrenia. In particular, a reliable neuroanatomical marker for the disease such as the DLPFC was chosen and the Bag-of-Words (BoW) paradigm was applied. The designed kernel was able to compare local regions localized in the DLPFC without imposing spatial constraint among them. Local features were encoded by multivariate descriptors which allowed for a greater versatility in capturing anatomical variations. The results were promising, since satisfactory scores were observed in the analysis of the whole dataset (up to 75\%), being higher when the subjects were stratified by sex and age $(84 \%$ for females and $81 \%$ for older subjects). Prior studies using automatic classification strategies for schizophrenia have reported similar rates, ranging between 75 and 90\% (Fan et al. 2011; Ince et al. 2008; Ingalhalikar et al. 2010; Pohl and Sabuncu 2009). In particular, Koutsouleris et al. (2009) have shown that pattern classification may be a valuable tool to detect psychosis event at the early stages of the illness. With regard to females with schizophrenia, prior MRI studies found increased probabilistic distribution of gray matter (Yoon et al. 2005) and higher classification accuracy (Fan et al. 2007). Similarly, older patients were better classify than younger individuals with schizophrenia. In this context, sexual dysmorphism and aging effects may have a significant interaction with the disease processes (Frazier et al. 2008; Granholm et al. 2000), possibly resulting in a better detection of the disease when SVM techniques are applied. Although age, chronicity, illness severity, and duration of treatment may in part explain male/female differences, in our sample men and women patient groups did not differ for those variables. Finally, the left hemisphere was classified with higher accuracy, which is consistent with prior pattern classification studies focused on cortical thickness (Yoon et al. 2007), potentially supporting the relevance of reduced laterality in schizophrenia (Bellani et al. 2009a, 2010b; Ribolsi et al. 2009). 
The DLPFC was used for brain classification in this study based on the evidence that it is a reliable anatomical marker of schizophrenia (Bellani et al. 2009b; LopezGarcia et al. 2006). In particular, the DLPFC (Broadmann areas 9 and 46) is part of the frontal-subcortical neural circuitry that modulates mood and emotional processing (Gray et al. 2002; Lopez-Garcia et al. 2006). It is connected with higher order association centres in the temporal and parietal lobe and is involved in working memory and executive functions (Cabeza and Nyberg 2000; Smith and Jonides 1999). As far as schizophrenia is concerned, several in vivo magnetic resonance imaging (MRI) and neuropsychological findings have clearly shown that DLPFC plays a crucial role in the pathophysiology of the disease (Cannon et al. 2005; Meyer-Lindenberg and Weinberger 2006). In particular, DLPFC may in part sustain deficits of working memory, context processing, and learning in schizophrenia (Brambilla et al. 2007, 2011; MacDonald and Carter 2003), which may be improved after cognitive training along with DLPFC activation (Haut et al. 2010). However, it should be mentioned that, although the DLPFC is a key structure (Glahn et al. 2005), it has clearly been shown that a complex neural network sustains the neurobiology of schizophrenia (Corradi-Dell'acqua et al. 2011; Kaymaz and van Os 2009) and that possibly different pathological processes may relate to particular subgroups (Fornito et al. 2009), based for instance on specific psychopathological dimensions (i.e. negative, positive, and disorganization symptoms) (Goghari et al. 2010). The use of combined ROI and SVM approaches might therefore consider other specific structural markers to further automatically characterize the disease, such as the anterior cingulate, the hippocampus, the superior temporal gyrus and the corpus callosum, which have consistently been found to be altered in schizophrenia (Baiano et al. 2007; Brambilla et al. 2005).

It should be noted that our findings may have partially been limited by the administration of antipsychotic drugs or by length of illness. However, no effects of the duration of treatment, dosages, or type of antipsychotics were found on the volumes of the DLPFC. Also, chronically ill and non-chronically ill patients had comparable DLPFC size. In regards of our tracing landmarks for detecting DLPFC, we followed the method suggested by (Prasad et al. 2005) which greatly includes Brodmann's areas 9 and 46. However, it should be considered that there is a large interindividual and inter-hemispheric anatomical variability in the DLPFC boundaries in humans (Rajkowska and Goldman-Rakic 1995b). Therefore, for some subjects part of other areas (i.e. 8 and 10) may possibly have been included in the tracing. The DLPFC indeed comprises the Brodmann's areas 9 and 46 predominantly but also a few transitional areas: 9-8, 9-45, 46-10, and 46-45
(Rajkowska and Goldman-Rakic 1995a). As a result, different landmarks and measurement procedures have generally been used (Crespo-Facorro et al. 1999; Sanches et al. 2009; Tisserand et al. 2002) and up to date there is still no "in vivo" gold standard technique to delimit this region in humans. In general, it should also be kept in mind that the role of SVM remains at the moment an ancillary diagnostic tool which tentatively improve the ability to diagnose patients with schizophrenia and that will probably never substitute the expertise of the clinical psychiatrist. Nonetheless, SVM techniques represent a very promising tool for clinical and research psychiatry and other sequences may be useful to further classify schizophrenia such diffusion weighted imaging or functional magnetic resonance imaging, as recently shown (Ingalhalikar et al. 2010; Ulas et al. 2011; Yang et al. 2010). Pattern classification has also satisfactory been used in other neuropsychiatric conditions, such as autism (Ingalhalikar et al. 2010) and Alzheimer's disease (Plant et al. 2010).

In conclusion, this study showed that the DLPFC can be used as a brain structural marker to detect subjects with schizophrenia using an integrated innovative ROI-SVM approach. Similar investigations should be carried out in first-episode patients, considering males and females separately since they may express differential patterns of DLPFC neuropathology.

Acknowledgments We thank Barbara Alberti for proofreading the manuscript. This work was partly supported by grants to Dr. Brambilla from the American Psychiatric Institute for Research and Education (APIRE), the Italian Ministry for University and Research, and the Italian Ministry of Health (IRCCS "E. Medea"). We acknowledge financial support from the FET programme within the EU-FP7, under the SIMBAD project (contract 213250).

Conflict of interest None.

\section{References}

Amaddeo F, Beecham J, Bonizzato P, Fenyo A, Knapp M, Tansella M (1997) The use of a case register to evaluate the costs of psychiatric care. Acta Psychiatr Scand 95:189-198

Amaddeo F, Burti L, Ruggeri M, Tansella M (2009) Long-term monitoring and evaluation of a new system of community-based psychiatric care. Integrating research, teaching and practice at the University of Verona. Ann Ist Super Sanità 45:43-53

American Psychiatric Association (1994) Diagnostic and statistical manual of mental disorders, 4th edn. The American Psychiatric Association, Washington

Andreone N, Tansella M, Cerini R, Rambaldelli G, Versace A, Marrella G, Perlini C, Dusi N, Pelizza L, Balestrieri M, Barbui C, Nose M, Gasparini A, Brambilla P (2007a) Cerebral atrophy and white matter disruption in chronic schizophrenia. Eur Arch Psychiatry Clin Neurosci 257:3-11

Andreone N, Tansella M, Cerini R, Versace A, Rambaldelli G, Perlini C, Dusi N, Pelizza L, Balestrieri M, Barbui C, Nose M, Gasparini A, Brambilla P (2007b) Cortical white-matter 
microstructure in schizophrenia. Diffusion imaging study. Br J Psychiatry 191:113-119

Arnone D, McIntosh AM, Tan GM, Ebmeier KP (2008) Metaanalysis of magnetic resonance imaging studies of the corpus callosum in schizophrenia. Schizophr Res 101:124-132

Ashburner J, Friston KJ (2000) Voxel-based morphometry-the methods. Neuroimage 11:805-821

Baiano M, David A, Versace A, Churchill R, Balestrieri M, Brambilla $P$ (2007) Anterior cingulate volumes in schizophrenia: a systematic review and a meta-analysis of MRI studies. Schizophr Res 93:1-12

Baiano M, Perlini C, Rambaldelli G, Cerini R, Dusi N, Bellani M, Spezzapria G, Versace A, Balestrieri M, Mucelli RP, Tansella M, Brambilla P (2008) Decreased entorhinal cortex volumes in schizophrenia. Schizophr Res 102:171-180

Bellani M, Marzi CA, Brambilla P (2009a) Interhemispheric communication in schizophrenia. Epidemiol Psichiatr Soc 18: $19-22$

Bellani M, Tomelleri L, Brambilla P (2009b) Emotion-based decision making in schizophrenia: evidence from the Iowa Gambling Task. Epidemiol Psichiatr Soc 18:104-106

Bellani M, Dusi N, Brambilla P (2010a) Longitudinal imaging studies in schizophrenia: the relationship between brain morphology and outcome measures. Epidemiol Psichiatr Soc 19:207-210

Bellani M, Marzi CA, Savazzi S, Perlini C, Cerruti S, Ferro A, Marinelli V, Sponda S, Rambaldelli G, Tansella M, Brambilla P (2010b) Laterality effects in schizophrenia and bipolar disorder. Exp Brain Res 201:339-344

Brambilla P, Cerini R, Gasparini A, Versace A, Andreone N, Vittorini E, Barbui C, Pelizza L, Nose M, Barlocco L, Marrella G, Gregis M, Tournikioti K, David AS, Keshavan MS, Tansella M (2005) Investigation of corpus callosum in schizophrenia with diffusion imaging. Schizophr Res 79:201-210

Brambilla P, Macdonald AW 3rd, Sassi RB, Johnson MK, Mallinger AG, Carter CS, Soares JC (2007) Context processing performance in bipolar disorder patients. Bipolar Disord 9:230-237

Brambilla P, Cerruti S, Bellani M, Perlini C, Ferro A, Marinelli V, Giusto D, Tomelleri L, Rambaldelli G, Tansella M, Diwadkar VA (2011) Shared impairment in associative learning in schizophrenia and bipolar disorder. Prog Neuropsychopharmacol Biol Psychiatry 35:1093-1099

Brurges C (1998) A tutorial on support vector machine for pattern recognition. Data Min Knowl Disc 2:121-167

Cabeza R, Nyberg L (2000) Neural bases of learning and memory: functional neuroimaging evidence. Curr Opin Neurol 13: 415-421

Cannon TD, Glahn DC, Kim J, Van Erp TG, Karlsgodt K, Cohen MS, Nuechterlein KH, Bava S, Shirinyan D (2005) Dorsolateral prefrontal cortex activity during maintenance and manipulation of information in working memory in patients with schizophrenia. Arch Gen Psychiatry 62:1071-1080

Corradi-Dell' acqua C, Tomelleri L, Bellani M, Rambaldelli G, Cerini R, Pozzi-Mucelli R, Balestrieri M, Tansella M, Brambilla P (2011) Thalamic-insular dysconnectivity in schizophrenia: evidence from structural equation modeling. Hum Brain Mapp (In Press)

Crespo-Facorro B, Kim JJ, Andreasen NC, O'Leary DS, Wiser AK, Bailey JM, Harris G, Magnotta VA (1999) Human frontal cortex: an MRI-based parcellation method. Neuroimage 10:500-519

Cruska, G, Dance CR, Fan L, Willamowski J, Bray C (2004) Visual categorization with bags of keypoints. ECCV Workshop on Statistical Learning in Computer Vision, pp 1-22

Davatzikos C, Shen D, Gur RC, Wu X, Liu D, Fan Y, Hughett P, Turetsky BI, Gur RE (2005) Whole-brain morphometric study of schizophrenia revealing a spatially complex set of focal abnormalities. Arch Gen Psychiatry 62:1218-1227
Duda RO, Hart PE, Stork DG (2001) Pattern Classification, 2nd edn. Springer, New York

Fan Y, Shen D, Gur RC, Gur RE, Davatzikos C (2007) Compare: classification of morphological patterns using adaptive regional elements. IEEE Trans Med Imaging 26:93-105

Fan Y, Gur RE, Gur RC, Wu X, Shen D, Calkins ME, Davatzikos C (2008) Unaffected family members and schizophrenia patients share brain structure patterns: a high-dimensional pattern classification study. Biol Psychiatry 63:118-124

Fan Y, Liu Y, Wu H, Hao Y, Liu H, Liu Z, Jiang T (2011) Discriminant analysis of functional connectivity patterns on Grassmann manifold. Neuroimage 56:2058-2067

Fornito A, Yucel M, Patti J, Wood SJ, Pantelis C (2009) Mapping grey matter reductions in schizophrenia: an anatomical likelihood estimation analysis of voxel-based morphometry studies. Schizophr Res 108:104-113

Frazier JA, Hodge SM, Breeze JL, Giuliano AJ, Terry JE, Moore CM, Kennedy DN, Lopez-Larson MP, Caviness VS, Seidman LJ, Zablotsky B, Makris N (2008) Diagnostic and sex effects on limbic volumes in early-onset bipolar disorder and schizophrenia. Schizophr Bull 34:37-46

Gerig, G, Styner, M, Shenton, ME, Lieberman, JA (2001): Shape versus size: Improved understanding of the morphology of brain structures. In: Medical image computing and computer-assisted intervention (MICCAI). LNCS, vol 2208, pp 24-32

Giuliani NR, Calhoun VD, Pearlson GD, Francis A, Buchanan RW (2005) Voxel-based morphometry versus region of interest: a comparison of two methods for analyzing gray matter differences in schizophrenia. Schizophr Res 74:135-147

Glahn DC, Ragland JD, Abramoff A, Barrett J, Laird AR, Bearden CE, Velligan DI (2005) Beyond hypofrontality: a quantitative meta-analysis of functional neuroimaging studies of working memory in schizophrenia. Hum Brain Mapp 25:60-69

Goghari VM, Sponheim SR, MacDonald AW 3rd (2010) The functional neuroanatomy of symptom dimensions in schizophrenia: a qualitative and quantitative review of a persistent question. Neurosci Biobehav Rev 34:468-486

Granholm E, Morris S, Asarnow RF, Chock D, Jeste DV (2000) Accelerated age-related decline in processing resources in schizophrenia: evidence from pupillary responses recorded during the span of apprehension task. J Int Neuropsychol Soc 6:30-43

Grauman K, Darrell T (2007) The pyramid match kernel: efficient learning with sets of features. J Mach Learn Res 8:725-760

Gray JR, Braver TS, Raichle ME (2002) Integration of emotion and cognition in the lateral prefrontal cortex. Proc Natl Acad Sci USA 99:4115-4120

Haut KM, Lim KO, Macdonald A 3rd (2010) Prefrontal cortical changes following cognitive training in patients with chronic schizophrenia: effects of practice, generalization, and specificity. Neuropsychopharmacology 35:1850-1859

Ince NF, Goksu F, Pellizzer G, Tewfik A, Stephane M (2008) Selection of spectro-temporal patterns in multichannel MEG with support vector machines for schizophrenia classification. Conf Proc IEEE Eng Med Biol Soc 2008:3554-3557

Ingalhalikar M, Kanterakis S, Gur R, Roberts TP, Verma R (2010) DTI based diagnostic prediction of a disease via pattern classification. Med Image Comput Comput Assist Interv 13: $558-565$

Kaymaz N, van Os J (2009) Heritability of structural brain traits an endophenotype approach to deconstruct schizophrenia. Int Rev Neurobiol 89:85-130

Kempton MJ, Stahl D, Williams SC, DeLisi LE (2010) Progressive lateral ventricular enlargement in schizophrenia: a meta-analysis of longitudinal MRI studies. Schizophr Res 120:54-62

Koutsouleris N, Meisenzahl EM, Davatzikos C, Bottlender R, Frodl T, Scheuerecker J, Schmitt G, Zetzsche T, Decker P, Reiser M, Moller 
HJ, Gaser C (2009) Use of neuroanatomical pattern classification to identify subjects in at-risk mental states of psychosis and predict disease transition. Arch Gen Psychiatry 66:700-712

Lopez-Garcia P, Aizenstein HJ, Snitz BE, Walter RP, Carter CS (2006) Automated ROI-based brain parcellation analysis of frontal and temporal brain volumes in schizophrenia. Psychiatry Res 147:153-161

Lowe DG (2004) Distinctive image features from scale-invariant keypoints. J Comput Vis 60:91-110

MacDonald AW 3rd, Carter CS (2003) Event-related FMRI study of context processing in dorsolateral prefrontal cortex of patients with schizophrenia. J Abnorm Psychol 112:689-697

Meyer-Lindenberg A, Weinberger DR (2006) Intermediate phenotypes and genetic mechanisms of psychiatric disorders. Nat Rev Neurosci 7:818-827

Plant C, Teipel SJ, Oswald A, Bohm C, Meindl T, Mourao-Miranda J, Bokde AW, Hampel H, Ewers M (2010) Automated detection of brain atrophy patterns based on MRI for the prediction of Alzheimer's disease. Neuroimage 50:162-174

Pohl KM, Sabuncu MR (2009) A unified framework for MR based disease classification. Inf Process Med Imaging 21:300-313

Potkin SG, Turner JA, Brown GG, McCarthy G, Greve DN, Glover GH, Manoach DS, Belger A, Diaz M, Wible CG, Ford JM, Mathalon DH, Gollub R, Lauriello J, O'Leary D, van Erp TG, Toga AW, Preda A, Lim KO (2009) Working memory and DLPFC inefficiency in schizophrenia: the FBIRN study. Schizophr Bull 35:19-31

Prasad KM, Sahni SD, Rohm BR, Keshavan MS (2005) Dorsolateral prefrontal cortex morphology and short-term outcome in firstepisode schizophrenia. Psychiatry Res 140:147-155

Prasad KM, Shirts BH, Yolken RH, Keshavan MS, Nimgaonkar VL (2007) Brain morphological changes associated with exposure to HSV1 in first-episode schizophrenia. Mol Psychiatry 12:105-113 (101)

Rajkowska G, Goldman-Rakic PS (1995a) Cytoarchitectonic definition of prefrontal areas in the normal human cortex: I. Remapping of areas 9 and 46 using quantitative criteria. Cereb Cortex 5:307-322

Rajkowska G, Goldman-Rakic PS (1995b) Cytoarchitectonic definition of prefrontal areas in the normal human cortex: II. Variability in locations of areas 9 and 46 and relationship to the Talairach Coordinate System. Cereb Cortex 5:323-337

Ribolsi M, Koch G, Magni V, Di Lorenzo G, Rubino IA, Siracusano A, Centonze D (2009) Abnormal brain lateralization and connectivity in schizophrenia. Rev Neurosci 20:61-70
Sanches M, Caetano S, Nicoletti M, Monkul ES, Chen HH, Hatch JP, Yeh PH, Mullis RL, Keshavan MS, Rajowska G, Soares JC (2009) An MRI-based approach for the measurement of the dorsolateral prefrontal cortex in humans. Psychiatry Res 173:150-154

Smith EE, Jonides J (1999) Storage and executive processes in the frontal lobes. Science 283:1657-1661

Tisserand DJ, Pruessner JC, Sanz Arigita EJ, van Boxtel MP, Evans AC, Jolles J, Uylings HB (2002) Regional frontal cortical volumes decrease differentially in aging: an MRI study to compare volumetric approaches and voxel-based morphometry. Neuroimage 17:657-669

Ulas A, Duin RPW, Castellani U, Loog P, Mirtuono P, Bicego M, Murino V, Bellani M, Cerruti S, Tansella M, Brambilla P (2011) Dissimilarity-based detection of schizophrenia. Int J Imaging Syst Technol 21:179-192

Ventura J, Nuechterlein KH, Subotnik KL, Gutkind D, Gilbert EA (2000) Symptom dimensions in recent-onset schizophrenia and mania: a principal components analysis of the 24-item Brief Psychiatric Rating Scale. Psychiatry Res 97:129-135

World Health Organisation (1992) Schedules for Clinical Assessment in Neuropsychiatry. WHO, Geneva

World Health Organisation (1996) In: Tansella M, Nardini M (eds) Schede di valutazione clinica in neuropsichiatria. SCAN 2.1. Il Pensiero Scientifico Editore, Roma

Yang H, Liu J, Sui J, Pearlson G, Calhoun VD (2010) A hybrid machine learning method for fusing fMRI and genetic data: combining both improves classification of schizophrenia. Front Hum Neurosci 4:192

Yoon U, Lee JM, Koo BB, Shin YW, Lee KJ, Kim IY, Kwon JS, Kim SI (2005) Quantitative analysis of group-specific brain tissue probability map for schizophrenic patients. Neuroimage 26: 502-512

Yoon U, Lee JM, Im K, Shin YW, Cho BH, Kim IY, Kwon JS, Kim SI (2007) Pattern classification using principal components of cortical thickness and its discriminative pattern in schizophrenia. Neuroimage 34:1405-1415

Yoon JH, Minzenberg MJ, Ursu S, Ryan Walter BS, Wendelken C, Ragland JD, Carter CS (2008) Association of dorsolateral prefrontal cortex dysfunction with disrupted coordinated brain activity in schizophrenia: relationship with impaired cognition, behavioral disorganization, and global function. Am J Psychiatry 165:1006-1014 